\section{Nicht-medikamentöse Therapieoptionen bei ADHS im Vergleich}

In einer Metaanalyse wurden verhaltenstherapeutisch-orientierte Therapien bei Patienten mit ADHS geprüft. Einige Methoden zeigten gute Ergebnisse.

— in Team von Wissenschaftlern be- wertete 14 kontrollierte Therapiestudien und verglich ihre Wirksamkeit untereinander. Erfasst wurden:

- Verhalten-modifizierende Therapien

— Neurofeedback

- multimodale psychosoziale Therapien

— schulbasierte Programme

_ Training des Arbeitsgedächtnis

_Elterntraining

_-Selbst-Monitoring-Programme.

Die Wirksamkeit auf die ADHS-Symptomatik bei 625 Studienteilnehmern (382 Therapieteilnehmer und 243 Kontrollen) wurde evaluiert. Als Parameter zur Beurteilung der Wirkung wurden die Conners Parenting Scales, die Conners' Teacher Rating Scales sowie die Beurteilung von Hyperaktivität, Unauf- merksamkeit, Impulsivität und Arbeitsgedächtnis herangezogen.

Die Autoren fanden die höchsten Effektstärken bei den Verhalten-modifizierenden Verfahren und beim Neurofeedback. Mädchen profitierten mehr als die Jungen von den Therapien; bei der kombinierten Form der ADHS zeigten sich die Therapien weniger effektiv als bei der rein unaufmerksamen Form. Das Ausmaß der Therapie hatte keinen Einfluss auf die Wirksamkeit.

Hodgson $\mathrm{K}$ et al. Non pharmacological treatments for ADHD: a meta-analytic review.

J Atten Disord 2012 May 29 [Epub ahead of print]

Kommentar: Wie auch schon die Autoren selbst angeben, wurde die Metaanalyse erschwert durch die inhomogenen Bewertungsmaßstäbe. Dennoch sollte das schlechte Abschneiden insbesondere der multimodalen psychosozialen Trainings und auch der Konzentrationstrainings, die auf eine Verbesserung des Arbeitsgedächtnisses abzielen, zu denken geben. Denn an diesen orientieren sich häufig die bei uns in Ergotherapien stattfindenden therapeutischen Maßnahmen.

Erstaunlich ist auch das schlechte Abschneiden des Elterntrainings. Möglicherweise spielt dabei eine Rolle, dass hier auch selbst von ADHS betroffene Eltern mit erfasst werden, bei denen studienbelegt die Wirkung des Trainings eingeschränkt ist. Das in der Metaanalyse am besten abschneidende Neurofeedback wird in Deutschland immer noch nicht von den Krankenkassen übernommen. Um den Kindern überflüssige, zeitraubende und vielleicht sogar schädliche Therapien zu ersparen, sollten in Deutschland die bestehenden nicht-medikamentösen Therapieangebote endlich seriös evaluiert werden.

Dr. Kirsten Stollhoff

\title{
Nach welcher Zeit sollte der Pricktest abgelesen werden?
}

Ein Pricktest ist in der allergologischen Diagnostik ein preiswertes, erprobtes und bewährtes Verfahren. Routinemäßig wird er üblicherweise nach 15 bis 20 Minuten abgelesen. Unklar ist aber weithin, wie stark die Schwankungen sein können, um trotzdem verlässliche Werte zu bekommen.

ie Frage nach dem idealen Zeitpunkt zur Auswertung eines Pricktests konnte nun ein amerikanisches Team um Sara Seibert vom College of Medicine der Penn State University in Pennsylvania klären: Dazu führten sie bei 57 Patienten in einer prospektiven Studie jeweils Pricktests mit unterschiedlichen AeroAllergenen durch und maßen dann nach 10, 20, 30 und 40 min die Testquaddel und das umgebende Erythem. Sie verglichen dann die Quaddeln und Erytheme, die

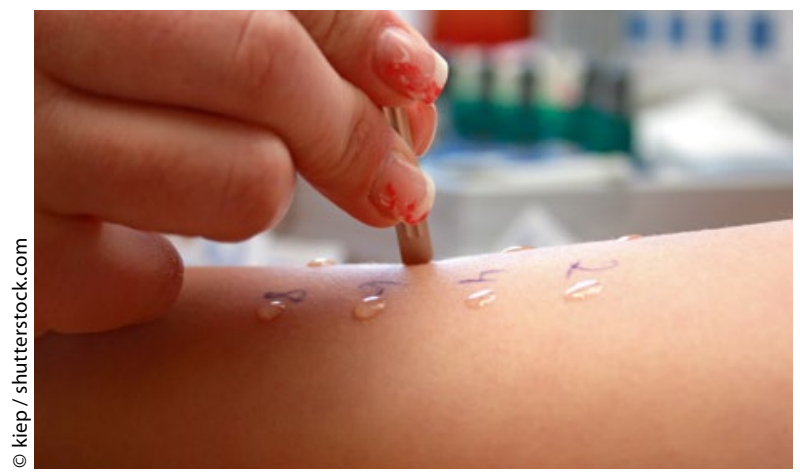

Mit 20 Minuten Wartezeit ist man beim Pricktest auf der sicheren Seite. nach der üblichen 20-min-Wartezeit auftraten, mit denen nach 10, 30 und $40 \mathrm{~min}$.

In der statistischen Auswertung sahen sie eine enge Korrelation der Flächen bei der 20- und 30-minütigen Ablesung, jedoch eine deutlich schwächere zu den Resultaten nach 10 bzw. 40 min. Bei noch kürzerer Ablesezeit (weniger als 10 min) und auch bei längerem Zuwarten als 40 min sank die Verlässlichkeit.

Seibert SM et al. Reliability of skin test results when read at different time points. Allergy Asthma Proc 2011; 32: 203-5

Kommentar: Die traditionelle Zeitspanne beim Ablesen des Pricktests mit 15 bis 20 min ist am besten geeignet für die korrekte Auswertung; sollten aber in der Praxis widrige Umstände den empfohlenen Ablesezeitpunkt verhindern, kann auch im Zeitraum von 10 bis 40 min nach dem Piks noch ein gutes und verlässliches Ergebnis erzielt werden. Dieses Resultat gibt den Praxen mehr Flexibilität. Zeiten unter 10 und über 40 min beinhalten aber das Risiko einer deutlich abnehmenden Qualität.

Dr. Ulrich Mutschler 